Conclusions Our study indicates that EUS FNA microcore biopsy is more sensitive than endobiliary biopsy in the diagnosis of malignant pancreaticobiliary lesions. Because lesions are visualised, sampling is targeted and this provides high tissue yield enabling a malignant histological diagnosis to be rendered and reduces the need for repeated sampling. The tissue sample is also amenable to immunohistochemical staining which is important in characterising suspected metastases. EUS FNA microcore biopsy has been demonstrated to be useful in sampling suspected primary biliary neoplasm. As such, we believe that EUS FNA should be the standard method of tissue sampling in suspected malignant pancreaticobiliary lesions.

\section{PTU-026 SURGICAL MANAGEMENT OF DUODENAL NEUROENDOCRINE TUMOURS}

Michail Pizanias*, Elissaios Kontis, Omar Barbouti, Evangelos Prassas, John Ramage, Andreas Prachalias, Rajaventhan Srirajaskanthan. King's College Hospital, London, UK

\subsection{6/gutjnl-2018-BSGAbstracts.311}

Introduction Duodenal neuroendocrine tumours (D-NETs) are rare tumours. The management of D-NETs is complex due to the lack of understanding of the natural course of disease. We present a single centre experience in the surgical management of these tumours with long term follow up.

Methods Retrospective single arm observational study of DNETs treated at our institution between January 2010 until August 2017.

Results Twenty four patients (13 male), with a mean age of $60.33 \pm 13.6$ were treated for D-NETs during the study period. The patients either underwent pancreaticoduodenectomy ( 7 patients-29.2\%), segmental duodenal resection (7 patients29.2\%) or Endoscopic Mucosal Resection (10 patients-41.7\%). The mean overall survival was $96.08 \pm 3.82$ months $(95 \% \mathrm{CI}$ : $88.58-103.58)$; 6 patients presented recurrence at $23.41 \pm 8.65$ months. There was no statistical significant difference in either disease free survival (Mantel-Cox Log Rank $\mathrm{p}=0.327$ ) or overall survival (Mantel-Cox Log Rank $\mathrm{p}=0.317$ ) between patients undergoing each type of resection. Among patients who underwent surgical resection (pancreaticoduodenectomy or segmental resection) we were unable to correlate the size of the tumour with presence of nodal disease at the time of resection. Among those patients who presented with metastatic liver lesions, those were of the same or lower grade compared to primary tumour.

Conclusions Formal oncological surgical resection should be considered in patients with locally advanced disease if patients are fit and wish to undergo surgery. In patients with localised disease (pT1) endoscopic resection can be considered with evidence of good long term survival.

\section{PTU-027 IS METABOLIC BONE DISEASE ROUTINELY TESTED FOR IN CHRONIC PANCREATITIS?}

Fahd Rana*, Noor Bekkali, Richard Charnley, Jennifer Logue, Manu Nayar, Kofi Oppong, John Leeds. Freeman Hospital, Newcastle Upon Tyne, UK

\subsection{6/gutjnl-2018-BSGAbstracts.312}

Introduction Chronic pancreatitis is associated with metabolic bone disease which increases the risk of fragility fractures. The National Institute of Clinical Excellence (NICE) guidance recommends that all patients aged 50 or over should be considered for DEXA scanning if at risk. Previous data has shown underutilisation of DEXA scanning in this population despite increased risk of osteoporosis. The aim of this study was to assess compliance with metabolic bone assessment in patients with chronic pancreatitis, assess the prevalence of abnormal DEXA scans and the impact of this assessment on appropriate management.

Patients and methods Retrospective analysis of outpatient coding for "chronic pancreatitis" was performed over a 2 year period. Patient demographics, aetiology of chronic pancreatitis, prescription of pancreatic enzyme replacement therapy (PERT), vitamin D levels, DEXA scan result, history of fractures and bone protection medications were noted. Univariate and multivariable analysis were performed to explore why DEXA scanning was not performed as well as factors associated with abnormal scans. The impact of DEXA scanning on prescription of bone protection was also assessed.

Results 134 chronic pancreatitis patients (mean age 57.6 years, 88 males) were included with aetiology recorded as alcohol $(n=68)$, idiopathic $(n=52)$, hypertriglyceridaemia $(n=5)$, autoimmune $(n=4)$, hereditary $(n=3)$, anatomical $(n=1)$ and biliary $(n=1) .102 / 134(76.1 \%)$ had vitamin D levels tested of which 82/104 (78.8\%) were low. 62/134 (46.3\%) had been sent for DEXA scanning of which 8 results were unavailable, 19 (30.6\%) were normal, 24 (38.7\%) showed osteopenia and 11 (17.7\%) osteoporosis. 46/62 (74.2\%) who had a DEXA scan were on bone protection compared to $30 / 72$ (41/7\%) who did not have a DEXA scan $(p=0.002)$. Lack of DEXA scanning was associated with female sex (adjusted OR 0.22, 95\% CI $0.09-0.57, \mathrm{p}=0.0017$ ) and not requiring PERT (adjusted OR $0.44,0.20-0.95, p=0.035)$. Not requiring PERT was also independently associated (protective) with abnormal DEXA scan results (adjusted OR 0.17, 95\% CI 0.03-0.98, p=0.047). 76 patients were prescribed bone protection with a higher proportion in those that had undergone a DEXA scan $(46 / 62$ with DEXA vs 30/72 without DEXA, p=0.002). 21/134 (15.7\%) had a previous fracture of which 10 had DEXA scanning. $8 / 10$ were on bone protection compared to 2/11 who had not had a

DEXA scan $(p=0.03)$.

Conclusions Despite a high prevalence of metabolic bone disease, less than half of chronic pancreatitis patients were assessed. Not requiring PERT and females were less likely to have a DEXA. Interestingly, DEXA scanning was associated with appropriate prescription of bone protection. Whether a standardised proforma would improve rates of metabolic bone assessments needs to be studied.

\section{PTU-028 LINKED COLOUR IMAGING INCREASES THE DIAGNOSTIC YIELD AND ACCURACY OF TYPE 1 GASTRIC CARCINOIDS}

Shraddha Gulati*, Rajaventhan Srirajaskanthan, Andrew Emmanuel*, John Ramage, Amyn Haji, Bu'Hussain Hayee. King's College Hospital, London, UK

\subsection{6/gutjnl-2018-BSGAbstracts.313}

Introduction Type 1 Gastric carcinoid tumours (GCTs) are the most common neuroendocrine tumours arising from enterchromaffin cell hyperplasia and hypergastrinaemia on a background of atrophic gastritis. Endoscopic diagnosis of Type 1 GCTs remains a challenge. White light endoscopy (WLE) and 